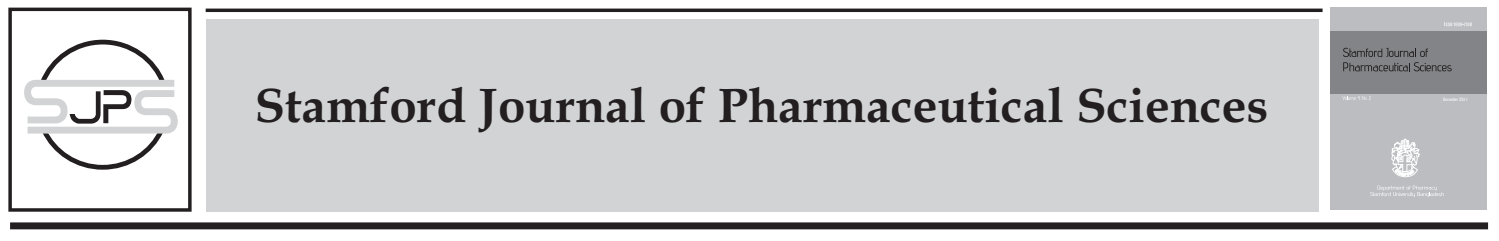

\title{
Assessment of dissolution profile of Pantoprazole tablets available in Bangladesh
}

\author{
*Aparajita Malakar, Bishwajit Bokshi, Utpal Kumar Karmakar \\ Pharmacy Discipline, Life Science School, Khulna University, Khulna-9208, Bangladesh
}

Brief Communication

\begin{abstract}
The aim of the present study was to increase the solubility of a poorly water soluble BCS class II drug, valsartan. Liquisolid technology and solid dispersion by kneading method were techniques used to improve the solubility of the drug by using non-volatile solvents and some hydrophilic carriers. Liquisolid compacts were prepared by dissolving the drug in suitable non volatile solvents. The various non volatile solvents used were PG, PEG, and glycerine. The carrier coating materials play an important role in improving the solubility of the drug. The dissolution rate of the drug was increased by using propylene glycol as non-volatile solvent at 20:1 ratio of carrier to coating material. Solid dispersion by kneading method were another attempt to improve solubility the various carrier materials used were PVP K 30, PEG 6000 and mannitol, these carriers are used in various ratios to improve its solubility. The dissolution rate of drug using solid dispersion kneading method with mannitol was increased at 1:3 ratio. The DSC and FTIR studies revealed no drug excipients interactions, whereas XRD revealed the reduced crystalinity of drug, which showed enhanced solubility. From the results it was concluded that the liquisolid compacts enhanced the solubility of valsartan in comparison to traditional solid dispersion method.
\end{abstract}

Keywords: Valsartan, liquisolid compacts, solid dispersions.

Pantoprazole is a group of drugs called proton pump inhibitor. It decrease the amount of acid produced in stomach (Drug Information, 2005).Pantoprazole is used to treat corrosive esophagitis (damage to the esophagus from the stomach acid), and other conditions involving excess stomach acid such as Zollinger-Ellison syndrome (Drug Information, 2005). The active ingredient in pantoprazole sodium delayed-release tablets is a substituted benzimidazole, sodium 5(difluoromethoxy)-2-[[(3,4-dimethoxy-2pyridinyl)methyl] sulfinyl]-1H-benzimidazole sesquihydrate, a compound that inhibits gastric acid secretion. Its empirical formula is

*Corresponding Author:

Aparajita Malakar

Student of M.Pharm, Pharmacy Discipline

Life Science School, Khulna University

Khulna-9208, Bangladesh

Contact No.: +880 1739370508

E-mail: aparajitamalakar@gmail.com
$\mathrm{C}_{16} \mathrm{H}_{14} \mathrm{~F}_{2} \mathrm{~N}_{3} \mathrm{NaO}_{4} \mathrm{~S} \times 1.5 \quad \mathrm{H}_{2} \mathrm{O}$, with $\mathrm{a}$ molecular weight of 432.4. Pantoprazole sodium sesquihydrate is a white to off-white crystalline powder and is racemic. Pantoprazole has weakly basic and acidic properties. Pantoprazole sodium sesquihydrate is freely soluble in water and very slightly soluble in phosphate buffer at $\mathrm{pH}$ 7.4.

The stability of the compound in aqueous solution is $\mathrm{pH}$-dependent. The rate of degradation increases with decreasing $\mathrm{pH}$. At ambient temperature, the degradation half-life is approximately 2.8 hours at $\mathrm{pH} 5.0$ and approximately 220 hours at $\mathrm{pH} 7.8$. (Current Medical Information, 2011).

Pantoprazole Sodium delayed-release tablet is official in USP (USP 30 and NF 25, 2009). An extensive literature survey reveals that many dissolution studies (Mansoor et al., 
2007; Campos et al., 2010; Chakraborty et al., 2009) on Pantoprazole Sodium delayedrelease tablet are reported. But there is no comparative study on commercially available pantoprazole tablets in Bangladesh. This study deals with the comparative in vitro dissolution characteristics of commonly available five brands of pantoprazole tablet in Bangladesh in order to find out any out of compliance market preparation.

Pantoprazole Sodium RS was obtained from Popular Pharmaceuticals Limited, Bangladesh. HPLC (Agilent 1100 series, USA) and Tablet dissolution tester (Electro Lab, India), Analytical balance (Shimadzu, Japan) was used for dissolution study. Five samples of different batches of marketed tablets were collected from various stores. The samples were properly checked for their manufacturing license number, batch number, manufacturing and expiry dates before purchasing. The samples were coded arbitrarily as S1, S2, S3, S4 and S5. The labeled active ingredient was pantoprazole 20 $\mathrm{mg}$ and packaged in blister packing. The blister packs were stored at $25 \pm 2^{\circ} \mathrm{C}$ for four weeks before the dissolution study in order to evaluate any change in organoleptic properties.

These studies were conducted at $37 \pm 0.5^{\circ} \mathrm{C}$ on an USP specification dissolution rate test type II apparatus (Paddle apparatus) with six sections assembly according to the USP dissolution test 1 (USP 32 and NF 27, 2009). $0.1 \mathrm{~N}$ hydrochloride acid was used as acid stage medium and phosphate buffer $(\mathrm{pH} 6.8)$ was used as buffer stage medium.

For preparing 32 liter of $0.1 \mathrm{~N}$ hydrochloride acid take 30 liter of water and mix $316.8 \mathrm{~mL}$ of hydrochloric acid (37\%). Finally, the volume was diluted to 32 liter to get $0.1 \mathrm{~N} \mathrm{HCl}$.

The water-bath temperature was fixed \& confirmed to be $37 \pm 0.5^{\circ} \mathrm{C}$ before starting the experiment. The medium was preheated to $37^{\circ} \mathrm{C}$ and then a quantity of $1000 \mathrm{~mL}$ was added to each vessel. The apparatus was then assembled and paddle rotation was started and adjusted at $75 \mathrm{rpm}$ and the system was allowed to equilibrate for 15 minutes. After that the paddle rotation was stopped and six tablets from same code were placed in the vessels (one tablet per vessel) and allowed to sink to the bottom. The apparatus was immediately operated at 75 rpm. The duration of the experiment was 2 hours for each set of sample. Five time points were selected to adequately characterize the ascending and plateau phases of the dissolution curve. $10 \mathrm{~mL}$ of sample solution was withdrawn after each 30 minutes. The withdrawn volume was immediately replaced by acid stage medium. The sample was filtered through $0.45 \mu \mathrm{m}$ filter and immediately was diluted a portion of the filtrate by a factor of 2 with $0.5 \mathrm{~N}$ sodium hydroxide. The resultant sample was analyzed by HPLC as directed for procedure

For the preparation of diluent a mixture of $\mathrm{pH}$ 6.8 phosphate buffer and $0.5 \mathrm{~N}$ sodium hydroxide (1:1) was prepared.

For the preparation of mobile phase a filtered and degassed mixture of water, acetonitrile, and triethylamine $(60: 40: 1)$ was prepared. Then $\mathrm{pH}$ of the mixture was adjusted with phosphoric acid to $7.0 \pm 0.05$.

For the preparation of standard solution 20 $\mathrm{mg}$ of pantoprazole sodium RS was accurately weighed and was transferred into $50 \mathrm{~mL}$ of volumetric flask. Then $30 \mathrm{~mL}$ of $0.02 \mathrm{~N}$ sodium hydroxide was added and was sonicated until dissolved. After that $2 \mathrm{~mL}$ of acetonitrile was added and was diluted with $0.02 \mathrm{~N}$ sodium hydroxide to volume. This was the mother solution. From the mother solution $0.4,0.6,0.8,1,1.2, \& 1.4 \mathrm{~mL}$ had been taken to $20 \mathrm{~mL}$ volumetric flask. Then the final volume was made to $20 \mathrm{~mL}$ with diluent. Thus the concentrations of this standard solution were $8,12,16,20,24$, \& $28 \mathrm{~g} / \mathrm{mL}$.

The liquid chromatograph was equipped with a $290-\mathrm{nm}$ detector and a $4.6-\mathrm{mm} \times 7.5-\mathrm{cm}$ column that contains $3-\mu \mathrm{m}$ packing $\mathrm{L} 1$. The column was maintained at $30^{\circ} \mathrm{C}$. The flow rate was about $1.0 \mathrm{~mL}$ per minute. Chromatograph the working standard solution, and record the peak responses as directed for procedure: the tailing factor was not more than 2.5, and the relative standard deviation for replicate injections was not more than $2.0 \%$.

Equal volumes (about $10 \mu \mathrm{L}$ ) of the working standard solution and the solution under test 
were separately injected into the chromatograph. Calculate the amount of pantoprazole released in the acid stage by the formula:

in which $r_{U}$ and $r_{S}$ are the peak responses of pantoprazole obtained from the solution under test and the Working standard solution, respectively; $\mathrm{C}_{\mathrm{S}}$ is the concentration, in $\mathrm{mg}$ per $\mathrm{mL}$, of pantoprazole sodium in the Working standard solution; 383.37 is the molecular weight of pantoprazole; 1000 is the volume, in $\mathrm{mL}$, of medium; 100 is the conversion factor to percentage; 405.35 is the molecular weight of pantoprazole sodium; and $\mathrm{L}$ is the tablet label claim, in $\mathrm{mg}$.

For preparing 32 liter buffer stage medium, $44 \mathrm{gm}$ sodium dihydrogen phosphate monohydrate and $36.8 \mathrm{gm}$ disodium hydrogen phosphate dihydrate were dissolved in distilled water and diluted to about 31.8 liter with the same solvent. Buffer $\mathrm{pH}$ was adjusted to 6.8 by adding dilute sodium hydroxide. Finally, the volume was diluted to 32 liter.

The water-bath temperature was fixed and confirmed to be $37 \pm 0.5^{\circ} \mathrm{C}$ before starting the experiment. The medium was preheated to $37^{\circ} \mathrm{C}$ and then a quantity of $1000 \mathrm{~mL}$ was added to each vessel. The apparatus was then assembled and paddle rotation was started and adjusted at $75 \mathrm{rpm}$ and the system was allowed to equilibrate for 15 minutes. After that the paddle rotation was stopped and six tablets from same code which already have passed acid stage medium were placed in the vessels (one tablet per vessel) and allowed to sink to the bottom. The apparatus was immediately operated at $75 \mathrm{rpm}$. The duration of the experiment was 30 minutes for each set of sample. Four time points were selected to adequately characterize the ascending and plateau phases of the dissolution curve. $10 \mathrm{~mL}$ of sample solution was withdrawn after each 10 minutes. The withdrawn volume was immediately replaced by buffer stage dissolution medium. The sample was filtered through $0.45 \mu \mathrm{m}$ filter and immediately was diluted a portion of the filtrate by a factor of 2 with $0.5 \mathrm{~N}$ sodium hydroxide. The resultant sample was analyzed using the same procedure as for the acid stage.

The calibration curve was found to be linear in the range of $8-28 \mathrm{~g} / \mathrm{ml}$ with regression equation of $y=2.5+48692.86 x ;\left(r^{2}=0.9999\right)$ which clearly indicates linearity of the method.

Drug release from each commercial samplewas calculated by taking the average reading of all corresponding sub-samples. The drug release in acid stage medium is summarized in Table 1.

Table 1: Drug release pattern from five commercial samples in acid stage medium under investigation.

\begin{tabular}{llllll}
\hline \multirow{2}{*}{$\begin{array}{l}\text { Time } \\
\text { (min) }\end{array}$} & \multicolumn{5}{l}{ Cumulative Percent Drug Release } \\
\cline { 2 - 6 } & S1 & S2 & S3 & S4 & S5 \\
\hline 0 & 0.2 & 0 & 0 & 0 & 0 \\
30 & 0 & 0 & 0 & 0 & 0 \\
60 & 0 & 1.48 & 0 & 2.11 & 0 \\
90 & 0 & 2.12 & 1.28 & 3.15 & 0 \\
120 & 0 & 3.42 & 4.51 & 5.16 & 0 \\
\hline
\end{tabular}

The in vitro release data (cumulative percent drug release) in the acid stage medium have been plotted against time to get a graphical presentation of the data (Figure 1). According to USP dissolution test 1 , not more than $10 \%$ of the labeled amount of pantoprazole should be dissolved in 2 hours. All the brands met the official standard under acid stage medium.

The drug release in the buffer stage dissolution medium is summarized in Table 2. The in vitro release data (cumulative percent drug release) in the buffer stage dissolution

Table 2: Drug release pattern from five commercial samples in buffer stage medium under investigation.

\begin{tabular}{llllll}
\hline \multirow{2}{*}{$\begin{array}{l}\text { Time } \\
\text { (min) }\end{array}$} & \multicolumn{6}{l}{ Cumulative Percent Drug Release } \\
\cline { 2 - 6 } & S1 & S2 & S3 & S4 & S5 \\
\hline 0 & 0 & 0 & 0 & 0 & 0 \\
10 & 52.35 & 41.87 & 60.18 & 45.87 & 75.4 \\
20 & 60.25 & 58.62 & 88.95 & 70.65 & 85.14 \\
30 & 64.23 & 94.47 & 92.75 & 81.25 & 87.56 \\
\hline
\end{tabular}

medium have been plotted against time to get a graphical presentation of the data (Figure 2). According to USP dissolution test 1 , not less than $75 \%(Q)$ of the labeled amount of pantoprazole should be dissolved in 30 


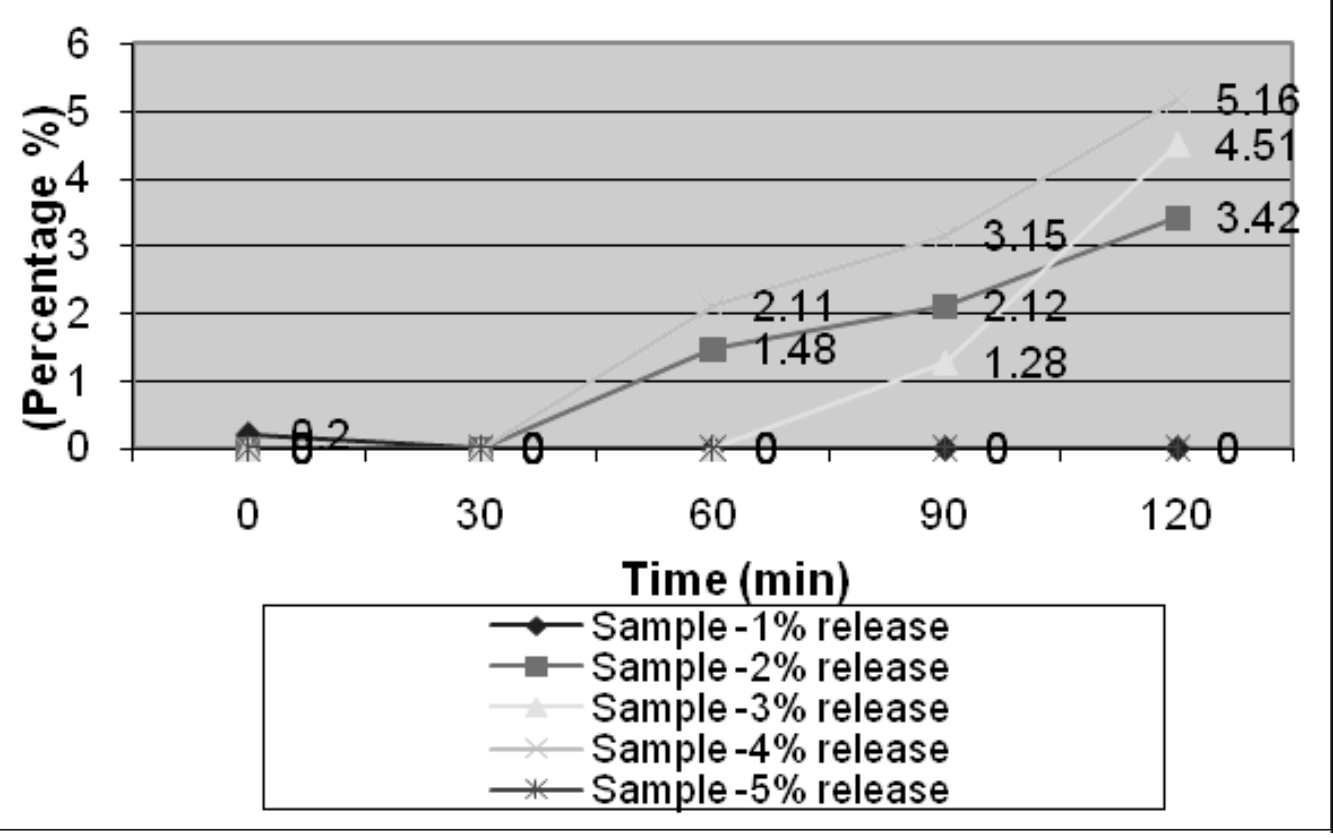

Figure 1: Comparison of release profiles of test samples in acid stage medium.

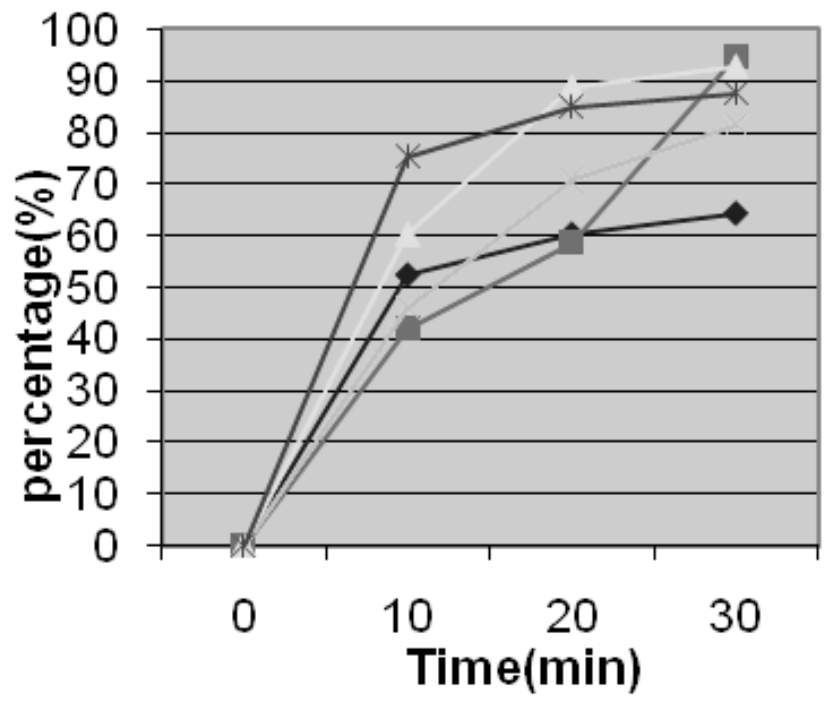

$\longrightarrow$ Sample $-1 \%$
release
$\rightarrow-$ Sample $-2 \%$
release
Sample $-3 \%$
release
- Sample $-4 \%$
release
$\rightarrow$ Sample $-5 \%$
release

Figure 2: Comparison of release profiles of test samples in buffer stage medium. 
minutes. Except one brand (Code: S1), all the brands met the official standard.

From this study it has been revealed that few commercially available brands of pantoprazole in Bangladesh failed to fulfill the official specification of dissolution test which might be explained by poor formulation and/or lower content of the active ingredient. This type of study should be performed more frequently and with more samples to build public awareness about the quality of marketed pharmaceutical products.

\section{REFERENCES}

Campos DR, Klein S, Zoller T, Vieria NR, Barros FA, Meurer EC et al. (2010). Evaluation of pantoprazole formulations in different dissolution apparatus using biorelevant medium. Arzneimittelforschung. 60(1):42-7. http://dx.doi.org/10.1055/s-0031-1296247

Current Medical Information. Available at: http://dailymed.nlm.nih.gov [Accessed on December 2, 2011].

Drug information (Available at: http://www.drugs.com) [Accessed on December 2, 2011].

Mansoor S, Sharma R, Dhakal S (2007). Comparative in vitro evaluation of commercially available pantoprazole tablets. Kathmandu University Journal of Science,

Engineering and Technology. 3: 1-7.

Chakraborty S, Sarkar S, Debnath SK (2009).

Formulation Development and Evaluation of Pantoprazole Enteric Coated Tablets. International Journal of ChemTech Research . 1: 663-666.

USP 30 and NF 25 (2007) CD-ROM. 